\title{
Los empresarios agrícolas y la contratación de temporeros: el caso de la Plana de Lleida
}

\author{
Anna Mata \\ Universitat de Lleida \\ annamata@geosoc.udl.cat \\ Agustí González \\ Universitat de Lleida \\ agustin.gonzalez@pip.udl.cat
}

Resumen: Este artículo analiza la organización que siguen los empresarios agricolas ilerdenses para llevar a cabo la contratación de empleados. A través de la realización de una encuesta durante el año 2015 y entrevistas a representantes de organizaciones agrarias antes $y$ después de dicha campaña, se ha recopilado información sobre sus actuaciones. Los resultados reflejan la planificación que siguen estos empresarios para realizar las contrataciones, los criterios que utilizan para decidir que trabajadores finalmente contratan y los perfiles de empleados que prefieren para cada tipo de actividad.

Palabras clave: Temporeros; contratación; mercado laboral agrícola; inmigración.

Abstract: This paper analyses how managers perform this enrollment. We did it through a survey during 2015's crop season and we made interviews to representatives of agrarian organizations before and after that campaign. The results reflects the planning that these entrepreneurs follow to make the hiring, the criteria they use to decide which workers they finally employ and the profiles of employees they prefer for each type of activity.

Key words: Seasonal workers; recruitment; agricultural labor market; immigration. 


\section{Introducción}

La agricultura intensiva basada en el cultivo de fruta para la exportación ha sido una de las actividades principales de las comarcas del sur de la provincia de Lleida. Esta actividad, aunque mecanizada desde los años 50-60 del siglo pasado, necesita de gran cantidad de mano de obra (de forma estacional), en los periodos de recolección, aclareo y poda de los cultivos.

Atendiendo a la distribución de la población activa en España (con menos del $5 \%$ del total ocupado en el sector agrícola ${ }^{1}$ ); a las condiciones del mercado laboral (caracterizado por alta precarización y bajada de salarios en términos absolutos); a la depreciación de las tareas agrícolas en la gama de posibilidades laborales del mercado de trabajo español y al hecho de que, en los últimos años, haya sido sobretodo población extranjera la que haya realizado las campañas de recolección, los empresarios agrícolas han tenido que esbozar diferentes estrategias para conseguir finalizar con éxito la campaña agrícola (que se inicia, según las zonas y los cultivos, en el mes de mayo y finaliza en el mes de septiembre-octubre).

El presente artículo pretende mostrar la organización de la campaña agrícola del año 2015, desde el punto de vista de los empresarios agrícolas y reflexionar sobre los entresijos, complejidades y contradicciones de la misma.

\section{Objeto de estudio y metodología}

En el estudio que hemos realizado en la "Plana de Lleida" pudimos detectar diferentes tipos de empresarios vinculados a la agricultura. Algunos de ellos manifiestan tener otros cultivos diferentes a la fruticultura o realizar otras actividades como la ganadería. Se trata de un colectivo, pese a existir en la zona, minoritario y que por sus características requieren menor cantidad de empleados.

Debido a los objetivos del mismo vamos a referirnos a partir de ahora a agricultores que su actividad principal es la fruticultura, aunque algunos de ellos, como veremos, tienen otras actividades agrícolas y ganaderas, en la mayoría de los casos complementarias y compatibles con la fruticultura.

Se ha sido configurado a partir de tres fases diferentes, una primera de entrevistas en profundidad a personas conocedoras de la campaña 2014 para analizar cómo se llevó a cabo, las situaciones que se vivieron, los puntos fuertes y débiles existentes en la organización y los factores incontrolables que existen.

La segunda fase de la investigación ha consistido en el diseño de un cuestionario y la realización de un trabajo de campo durante los meses de la campaña agrícola del año 2015. Con el fin de definir los perfiles de los empresarios y sus

1 Datos de INE (2016). 
preferencias en la contratación de temporeros. La muestra ha sido de 150 personas que dan trabajo de alguna manera a temporeros durante la temporada agrícola del verano de 2015.

Realizado el trabajo empírico, se ha hecho un análisis estadístico de resultados utilizando pruebas de significación estadística y los principales resultados se presentan en el siguiente artículo.

Por último, la tercera fase de la investigación parte de las dos anteriores y ha replicado entrevistas en profundidad de la fase uno para obtener una visión de la campaña agrícola 2015. Es decir, se ha profundizado en la valoración y los puntos fuertes y débiles para poder colaborar a mejorar la campaña 2016 y las siguientes.

\section{La agricultura en la Plana de Lleida}

\subsection{Contexto socioeconómico}

España ha sido un país históricamente muy vinculado a la actividad agrícola, tanto es así, que esta actividad no perdió su estatus de principal sector empleador hasta la década de los 70 del siglo pasado. Fue en ese período cuando se consiguió una fuerte recuperación económica motivada por la apertura a los mercados exteriores, el aumento de la inversión pública, la construcción de infraestructuras y la aparición de España como destino turístico internacional (Requena, 2008). Para la agricultura española fue el inicio del primer gran éxodo rural, iniciando definitivamente el declive del sector primario en pos de puestos más atractivos en las crecientes industrias de las ciudades o en el sector servicios vinculados con el comercio urbano o las zonas turísticas. Esta situación fue consolidada finalmente por la entrada de España en la Unión Europea y su afianzamiento como economía del sector servicios (Requeijo González, 2005).

Así, como observamos en la Tabla 1, el sector agrícola, principal sector ocupacional en España hasta los años 70; desciende hasta situarse por debajo del 5\% del total de población activa del país.

La Tabla nos muestra también el notable incremento de población activa de los últimos sesenta años de nuestra historia, a la par del incremento sostenido del sector servicios (que ocupaba en 2015 al 65,25\% de la población activa). En opinión de López y Rodríguez (2010) este incremento pone cifras a la consolidación del modelo desarrollista en nuestro país; modelo basado en la potenciación de la construcción y el turismo como modelo socioeconómico. 
Tabla 1. Distribución porcentual de la población activa por sectores de actividad, 1950-2015

\begin{tabular}{|c|c|c|c|c|c|c|}
\hline Año & $\begin{array}{c}\text { Total población } \\
\text { activa (Miles) }\end{array}$ & $\begin{array}{c}\text { Agricultura, } \\
\text { caza y pesca }\end{array}$ & Industria & Construcción & Servicios & Otros \\
\hline 1950 & $10.375,2$ & 47,6 & 19,9 & 6,6 & 25,9 & 0 \\
\hline 1960 & $11.235,1$ & 36,6 & 23,5 & 6,7 & 33,2 & 0 \\
\hline 1970 & $11.595,7$ & 22,8 & 27,9 & 10,5 & 38,8 & 0 \\
\hline 1980 & $10.492,5$ & 13,9 & 28,4 & 8,8 & 48,9 & 0 \\
\hline 1990 & $12.195,0$ & 8 & 25,5 & 11,3 & 55,2 & 5,5 \\
\hline 2000 & $20.885,70$ & 5,3 & 16,5 & 12 & 62,3 & 3,9 \\
\hline 2010 & $23.364,60$ & 4,28 & 12,48 & 9,22 & 64,56 & 9,46 \\
\hline 2015 & $22.922,00$ & 4,32 & 11,63 & 5,59 & 65,25 & 13,21 \\
\hline
\end{tabular}

Fuente. Instituto Nacional de Estadística (2016). Elaboración propia

Este modelo, junto con las mejoras en la formación y cualificación laboral, favorecerá la tendencia a ocupar puestos en la industria y los servicios, en detrimento del sector agrícola. Aunque, en opinión de Gordó et alii (2015), fueron también las condiciones de trabajo que ofrecía el sector agrícola: carácter cíclico, menor consideración social y sueldos más bajos; las que acarrearon que, a principios de los años noventa del pasado siglo, exista una carencia de trabajadores para atender las diversas tareas generadas en el campo, en particular las más intensivas en el uso de mano de obra (como la recolección de cosechas).

Los años de bonanza económica significaron, por una parte, la consolidación del trasvase de población activa del sector primario al secundario y terciario, por una parte; $y$, asimismo, la consolidación de España (en especial las provincias de Huelva y Lleida) como productor y exportador hortofrutícola de primer orden.

Esta condición de productor y exportador conllevó, sin embargo, la instauración de una economía de escala basada en la pervivencia de ocupaciones de alta estacionalidad, baja especialización y bajos salarios. En el caso de la producción agrícola que más abunda en Lleida, las frutas requieren de una extracción manual que implican grande números de contrataciones. $\mathrm{Al}$ mismo tiempo, el hecho de que los cultivos estén limitados a pocas especies implica que la ocupación, mayoritariamente, sólo se producirá en los momentos de poda y recolección, que tienen una duración de pocos meses.

La población activa, en la medida en que podía acceder a ocupaciones de menor estacionalidad, menor dureza y mejores salarios, dejaba estos puestos laborales sin cubrir o los abandonaba sin terminar su vigencia contractual (Torres, 2013) 
Es aquí donde surge la necesidad de contratar a un tipo de trabajador muy concreto, uno que sólo trabaje durante este periodo de tiempo y después abandone el territorio para buscar un trabajo similar en otra zona. Estos trabajadores son los que hoy en día conocemos como temporeros.

\subsection{El inmigrante internacional como temporero}

En sus trabajos en Cáritas, a inicios de la década de los noventa, Tabares (1990) ya empezó a detectar esta realidad. Él se refiere a los temporeros como jornaleros que abandonan su lugar de residencia habitual para buscar trabajo en otro sitio de forma temporal, ya que en ese otro lugar no hay braceros suficientes para cubrir esos puestos de trabajo. Lo hace de la siguiente manera:

[...] aquel jornalero o jornalera que, al no encontrar trabajo en su tierra se ve obligado/a a salir de su casa para sobrevivir en temporadas ocasionales allí donde se necesiten braceros para faenas que no pueden o no quieren realizar los propios del lugar. (Tabares, 1990, pp.10)

Por su parte, Pedreño (1998), hace referencia a que un temporero ha de estar necesariamente vinculado con la categoría agrícola:

[...] al proceso de trabajo agrícola (cultivo y recolección) y al de manufacturación del producto en el almacén (o en el propio campo). (Pedreño, 1998, pp.55)

Tradicionalmente, el trabajo agrícola había recaído en el payés ${ }^{2} y$ su familia, quienes labraban sus campos a lo largo del año y sus ingresos provenían de las diferentes producciones que generaban y vendían en los mercados locales. La llegada de la agricultura intensiva y su ya mencionada especialización y corta duración, implicaron que no fuera suficiente una familia para conseguir la cosecha e hiciera falta la contratación de temporeros.

A finales de los años ochenta y principios de los noventa, la producción se llevaba a cabo con la colaboración de las familias del propietario más la contratación de otras familias provenientes de diferentes puntos del estado español y trabajadores especializados de la zona (Achón, 2010).

A partir de los años noventa, España consolida su economía basada en los servicios e inicia un periodo de crecimiento económico que duraría hasta la crisis del año 2008 (Català Rubio, 2013), lo cual incide en una reestructuración del sector agrícola, especialmente en zonas como el sur de Lleida en que se puede intensificar la producción frutícola.

2 El término payes proviene del catalán Pagès, que hace referencia a un campesino de Cataluña o de las Islas Baleares. Es comúnmente utilizado por los temporeros para referirse a sus empleadores. 
En esta etapa de la historia reciente española se produjo un cambio generacional, los hijos de los payeses se habían formado y prefirieron adentrarse, mayoritariamente, en otros sectores económicos que ofrecían mejores sueldos y mayor estabilidad. Esta mejora de las condiciones económicas también redujo el interés de las familias de jornaleros de comunidades autónomas como Andalucía o Extremadura, quienes preferían combinar trabajos ocasionales en sus respectivas zonas antes que trasladarse por un puesto de corta duración y con sueldo bajo (Márquez Domínguez, 2009).

El sector agrícola se encontró en esos momentos con serios problemas para conseguir trabajadores que ocuparán puestos de trabajo inestable y escasamente pagados ${ }^{3}$. Curiosamente, en estas mismas fechas también se produjo la consolidación de la inmigración internacional en España, que si bien ya había empezado años atrás, es en esta etapa cuando se reafirma. De esta manera, los inmigrantes extranjeros encontraron en los puestos vacantes del campo un nicho de empleo rápido que les permitió acceder a su primer fuente de ingresos en territorio español o, en otros casos, complementar los trabajos esporádicos hechos en otros momentos del año (Díaz Diego, 2009).

\subsection{Los sindicatos de empresarios agrarios en las contrataciones}

La vinculación del fenómeno de inmigración internacional y el trabajo temporero fue tal que comenzaron a hacerse habituales los casos de abusos por parte del empresariado hacia el más vulnerable colectivo de trabajadores extranjeros. Ante esta situación, muchos países europeos que también tenían temporeros trabajando en sus campos, como el Reino Unido o Francia, decidieron comenzar a desarrollar programas públicos para la gestión del fenómeno. Sin embargo, el caso español fue distinto y fueron las organizaciones de empresarios agrarios los que tomaron la iniciativa. De tal forma, estas agrupaciones de empresarios comenzaron a llevar a cabo experiencias de gestión descentralizadas a nivel local (López-Sala et alii, 2016).

Así fue como los sindicatos agrarios comenzaron su alta implicación en la gestión de las campañas agrícolas. El primer paso se dio, precisamente, en Cataluña, cuando uno de estos sindicatos ofreció un servicio de intermediación laboral para la contratación de inmigrantes mientras que prohibía a sus afiliados contratar extranjeros en situación legal irregular. Posteriormente, esta misma organización, comenzaría la construcción de redes de alojamientos para temporeros que iría

3 Según afirman los empresarios agrícolas en el trabajo de Allepuz, Torres y Gordo (2013), subir los sueldos para intentar atraer a los trabajadores que han abandonado el campo no es una opción. Debido a los reducidos precios de venta del producto y el escaso poder de control que tienen sobre éste, los payeses defienden que mantener los salarios bajos son la única vía para asegurar la rentabilidad de las explotaciones agrícolas. 
ampliándose paulatinamente por las comarcas agrícolas. Su consolidación llegó con la firma de acuerdos para una gestión conjunta entre este mismo sindicato de empresarios y los ayuntamientos donde se ubicaban los albergues (Gordo Márquez et alii, 2015).

El crecimiento económico español durante los primeros años del siglo xxI provocó que las experiencias de bajas en el campo por mejores oportunidades en otros sectores se repitieran una vez más. Ahora eran los inmigrantes los que abandonaban los trabajos de temporeros y dejaban una vez más a las explotaciones agrarias con escasez de mano de obra. Los empresarios agrícolas tuvieron que buscar nuevas estrategias de contratación y las encontraron en las contrataciones en origen, donde una vez más los sindicatos de empresarios llevaron la iniciativa (Achón, 2010).

En el momento de escribir este artículo, nos encontramos ante un nuevo contexto que, previsiblemente, cambie la forma de funcionar de las campañas otra vez. La crisis económica reciente ha disparado los índices de paro y aumentado las personas deseosas de buscar un trabajo en cualquier sector.

Es en esta situación donde se plantea el estudio. Se buscará saber cómo se organiza, a día de hoy, las contrataciones; qué criterios usan para hacer su selección; qué perfiles prefieren; conocer si hacen algunas actuaciones para garantizar unas mínimas condiciones entre sus empleados y qué proponen para mejorar la realidad agrícola ilerdense.

\section{Principales resultados}

\subsection{Los sindicatos agrarios ilerdenses y la planificación de la campaña}

La mayoría de los agricultores encuestados (64\%) forman parte de alguna organización de empresarios de la agricultura. Las organizaciones a las que pertenecen son la Unió de Pagesos, ASAJA y Cooperativas Locales, en orden descendente. Las Cooperativas Locales juegan un papel importante en la comercialización de la fruta y para proveerlos de distintos inputs (abonos y herbicidas, principalmente) necesarios para el proceso productivo. También les proporcionan servicios técnicos y de control relativos al correcto uso de dichos inputs (épocas de tratamiento fitosanitario, proporciones de los productos, etc.). Los dos sindicatos, además de ser organizaciones que defienden sus intereses, también facilitan la gestión de determinados servicios administrativos con distintas agencias (declaraciones de renta, planes de mejora, etc.). 
Dentro del grupo de personas entrevistadas, hemos seleccionado a aquellas que representaban a alguna de estas organizaciones agrícolas para poder reflejar como éstas preparan las campañas.

Las campañas de fruta, según afirman los representantes, son procesos complejos donde participa una gran diversidad de personas y organizaciones, las cuales hacen una amplia gama de actividades. El resultado de toda esta amalgama de interventores e intervenciones, sumado al gran impacto económico que tiene sobre el territorio y sobre los empleadores, hace que todos estén de acuerdo en que es necesario que exista una esmerada planificación antes de comenzar la recogida de la fruta. Esta práctica se empezó a sistematizar para poder resolver todos los cambios que emergían por la llegada de los temporeros.

La planificación destaca, de acuerdo a las personas entrevistadas, por un elevado grado de estructuración. Esto se refleja en una delimitación de los diferentes momentos de la campaña que, a su vez, van acompañados de unas actuaciones conjuntas de los actores que forman parte de la acogida de temporeros durante la campaña.

Todos los entrevistados hacen énfasis en que esta estructura es el punto más importante de toda la organización y, a la vez, lo que evita daños ante las muchas posibles situaciones que se pueden dar a lo largo del verano.

Uno de los entrevistados recuerda que cuando él comenzó a trabajar en el sector hace 25 años, en Lleida había campamentos de hasta 1.500 personas y, ahora, pese a que aún existen, se han visto reducidos y no suman más de 50 personas. Según él, este tipo de situaciones se han reducido gracias a la buena planificación que se hace en la Plana de Lleida desde hace años.

La organización de la campaña en sí, se hace en el mes de febrero, porque estamos hablando de que en Lleida damos alrededor de 25.000 puestos de trabajo (...) y, por tanto, requiere de mucho trabajo y muchos esfuerzos durante muchos meses de preparación, porque cuando llega el grueso importante de población se tenga todo muy organizado y no haya ningún problema. (Representante sindical 1)

La característica más destacada por todos los entrevistados en relación con el mejor aspecto de la campaña es la gran y buena preparación que se hace de la misma. Este hecho es visto como el elemento más positivo para todos, ya que esta profesionalidad asegura el éxito de las campañas.

La misma organización es buena, funciona bien. Esto es lo mejor que tenemos, que casi funciona sola. (Representante sindical 2) 
A partir de este elevado nivel de preparación, todas las personas con las que hemos podido hablar hacen algún tipo de actuación en diferentes situaciones en relación a la campaña. Estas, a su vez, se pueden dividir en tres etapas: precampaña, durante la campaña y post campaña.

\subsubsection{La precampaña en la Plana de Lleida}

Se trata del punto de partida de todas las campañas. Tiene lugar a principios de año, aproximadamente en el mes de febrero, cuando todas aquellas personas con algún tipo de vínculo con la fruta hacen acciones previas para hacer posible la producción de la fruta desde sus diferentes campos de actuación.

Los sindicatos de empresarios ofrecen ayudas a sus afiliados para conseguir los trabajadores necesarios que hagan posible la campaña de fruta de cada explotación. Pese a que el mecanismo más habitual, según los representantes sindicales, es el contacto directo entre empresario y trabajador.

Así, aproximadamente en el mes de abril, cuando el empresario agrícola ya puede hacer una previsión de los trabajadores que necesitará, llama directamente a los contratados en años anteriores para decir qué días se empieza, o bien, son los propios trabajadores los que contactan si todavía no tienen noticias para este mes.

Sin embargo, cuando se da la situación que el empresario no puede cubrir sus vacantes de puestos de trabajo con temporeros repetidores; ya sea por malas experiencias, por la negativa de los trabajadores a volver o por necesidad de ampliar la plantilla; es cuando el empresario puede dirigirse a los sindicatos agrarios para que le ayuden a encontrar un trabajador, puesto que todos los sindicatos agrarios disponen de algún tipo de bolsa de trabajo, gestionada por ellos mismos, a la que recurren para ocupar los puestos que les piden.

El primer paso en la organización de la contratación a través de la bolsa es hacer reuniones en los pueblos para prever la cantidad de ofertas que se harán públicas. Los representantes sindicales se dirigen a sus afiliados interesados en contratar para saber la cantidad de trabajadores que necesitarán, los perfiles que prefieren y las fechas para empezar y acabar el contrato.

Por parte de los trabajadores, si están interesados en trabajar en empresas vinculadas con el sindicato agrario, deben dirigirse a alguna de las delegaciones que tenga la organización, dar su currículo, sus datos y las informaciones que sean necesarias como estudios o experiencia. Se recoge toda esta información y se les introduce en la bolsa.

Una vez hecho esto, el funcionamiento se basa en la oferta-demanda, según los puestos de trabajo que haya, se van seleccionando los postulantes en la 
medida que cumplan los requisitos que pide el empresario. Una vez se cumplan los requisitos, se ponen en contacto el empleador y el trabajador, que es cuando acuerdan cómo será su relación laboral.

Otro servicio de contratación ofrecido por estas entidades durante esta etapa son las contrataciones en origen. Si bien es cierto que es una modalidad cada vez menos usada, sigue existiendo en las tierras ilerdenses, pero con un mínimo impacto.

No llega ni al 1\% del total que hacemos (la contratación en origen). (Representante sindical 2)

\subsubsection{Durante la campaña}

A finales de mayo o a principios del mes de junio, se inicia lo que se conoce como la campaña de la fruta propiamente dicha. Todo este proceso durará hasta, aproximadamente, el mes de septiembre. Durante este tiempo, si bien es cierto que la planificación ya ha sido llevada a cabo, los diferentes actores continúan realizando una serie acciones en relación a las contrataciones.

Una vez iniciada la campaña, el papel de los sindicatos de empresarios agrarios pasa a ser el de controlar e intentar cubrir posibles imprevistos en las plazas de trabajo que los empresarios puedan tener para ofertar.

En este periodo, la función habitual de los sindicatos agrarios durante la campaña es la de cubrir puestos de trabajo dados por bajas de trabajadores o por subidas repentinas de trabajo que hay en algunos momentos del verano. Para llevar a cabo esta tarea, utilizan las bolsas de trabajo y seleccionan la gente que aún no ha encontrado un empleo en la campaña y se encuentra en una de las comarcas frutícolas de Lleida.

Dentro de estos trabajos muy puntuales, se procura que la persona que ha sido elegida repita en otras explotaciones agrarias o almacenes que también busquen ocupar puestos de inmediato y durante un muy breve periodo de tiempo. Así, son un núcleo reducido de personas las que se encargan de ocupar puestos de urgencia en todas las explotaciones de la región y se consigue una relativa estabilidad pese a su condición.

\subsubsection{La postcampaña}

Una vez llegan los meses de septiembre y octubre, se suele dar por terminada la campaña de recolección de la fruta y, con ella, se produce la marcha de la mayor parte de los trabajadores que llegaron a la provincia durante los meses de verano.

Los representantes sindicales entrevistados coinciden en la importancia de establecer un sistema de circulación de trabajadores en todo el estado. El objeti- 
vo es conseguir que los trabajadores que vienen a hacer la temporada en Lleida puedan sumar la mayor cantidad de meses trabajados y, así, conseguir una cierta estabilidad dentro de la inestabilidad inherente al sector agrario.

Aplicando este sistema, se puede conseguir que un trabajador pueda llegar a tener entre seis y siete meses de trabajo ininterrumpidos.

De esta manera, los trabajadores van pasando por los diferentes cultivos de la provincia de Lleida y, más tarde, van hacia otras provincias de Cataluña, lo que consigue la concatenación.

Este circuito de trabajadores dentro del territorio catalán es posible gracias a las delegaciones de las centrales sindicales en toda Cataluña, donde se sigue el mismo sistema de colocación a partir de bolsas de trabajo en cada comarca. Actuación que se hace extensible al conjunto del estado con aquellos sindicatos que tienen delegaciones fuera de las comarcas catalanas.

De igual forma, los sindicatos agrarios que tengan delegaciones en otros lugares de España o estén asociadas a una federación de sindicatos pueden hacer lo mismo para todo el Estado español.

\subsection{Estructura y funcionamiento de las explotaciones de la Plana de Lleida}

Prácticamente todos los agricultores encuestados (94\%) son propietarios de las explotaciones que cultivan. Además, excepto en algunas de las grandes explotaciones que hemos abordado, realizan directamente las diferentes funciones de gestión, lo cual corrobora que no disponen de una estructura administrativa y de gestión, siendo ellos mismos, o a través de sus organizaciones empresariales o con la ayuda de gestorías, los que realizan el seguimiento económico y administrativo de sus explotaciones. Es lógico suponer que además reciben ayudas de técnicos agrícolas especializados en determinados momentos, para la gestión y planificación de ciertas actividades agrícolas, como puede ser, la aplicación de tratamientos fitosanitarios, de herbicidas y el control de los residuos de estas acciones. También tienen la ayuda de las organizaciones empresariales y de las cooperativas, así como de los técnicos de estas asociaciones, en determinadas situaciones que requieren asesoramiento especializado, lo cual encaja con la débil estructura administrativa y de gestión de sus explotaciones, especialmente en las de menor tamaño.

La actividad principal de los agricultores encuestados (98\%) es la producción de fruta. Su actividad se complementa con la de los encuestados de la industria agroalimentaria, que se dedican principalmente a la distribución de la fruta (86\%), a su transformación (43\%) y al empaquetado de fruta (14\%). Todo ello 
pone en evidencia las diferencias y complementariedades que existen en el sector frutícola, puesto que los agricultores son los productores de la fruta, y dejan en manos de las cooperativas o de la industria agroalimentaria las funciones de manipulación y distribución de lo que producen, hasta que el producto llega a la mesa de los consumidores.

La mayoría de los agricultores encuestados diversifican su producción y, aunque sea la fruticultura su actividad principal, la complementan con otros cultivos, que no requieren tanta mano de obra y que permiten ser mecanizados, por lo que sirven para complementar y rentabilizar las inversiones en maquinaria agrícola.

El sector frutícola en los últimos años ha sufrido una crisis que le lleva a una permanente reestructuración, debido al estancamiento y caída de los precios, los problemas de exportación, y los cambios en las variedades de tipo de frutales, que están produciendo (en el futuro se verá más claro) cambios constantes en el sector, que inciden entre otros aspectos en el tamaño de las explotaciones y, que conducen a un constante arranque y a replantar nuevas variedades que tienen más demanda en el mercado global. Esto requiere una fuerte capitalización de las explotaciones y produce miedos sobre su continuidad y viabilidad, ya que los agricultores tienen dudas permanentes sobre cuál debe ser el tamaño ideal de sus explotaciones, para que estas sean rentables, y sobre cuáles deben ser las variedades que los harán más competitivos en un sector expuesto a constantes cambios y modificaciones varietales. El asesoramiento que puedan recibir tal vez no sea suficiente para la aventura que inician cada vez que se ven obligados a arrancar las variedades que no son, o han dejado de ser demandadas o valoradas por el mercado, $y$ son pocos, $y$ muchas veces por poco tiempo, los agricultores que se adaptan o escogen las variedades adecuadas para la cambiante demanda ya que constantemente aparecen y desaparecen modalidades varietales.

Preguntados respecto el número de hectáreas que tiene cada explotación agrícola, vemos una gran variedad de situaciones, puesto que aunque la mediana del tamaño de las explotaciones ronda las 30 hectáreas, la desviación típica se sitúa en las 39 hectáreas. En cuanto a las hectáreas que dedican a la producción frutícola, la inmensa mayoría no llega a las 25 hectáreas, hay un grupo importante con menos de 14, y una minoría con explotaciones de grandes dimensiones. Esta distribución es el reflejo del proceso que ha sufrido el sector en las últimas décadas, que ha conducido a la desaparición de pequeñas explotaciones de menos de 10 hectáreas, y a la creación de explotaciones mayoritariamente entre las 15 y 25 hectáreas, mediante la compra o el arrendamiento de las explotaciones que por jubilación o abandono del sector quedaban libres. A la vez, han surgido explotaciones de gran tamaño que ya no responden al modelo de producción familiar, 
fundamentado en la fuerza de trabajo de la familia del productor que en momentos de intensidad de demanda de mano de obra la contrataban temporalmente.

Todas las explotaciones, independientemente de su tamaño, han llevado a cabo una importante mecanización de las tareas que requerían más fuerza de trabajo, no obstante todas requieren, a pesar de ello, de la contratación de temporeros en momentos determinados del ciclo productivo, aunque algunas tengan contratados trabajadores durante todo el año.

Las explotaciones de más de 25 hectáreas tienen mayor número de trabajadores a lo largo del año y en momentos como la poda, aclareo y recogida de la fruta también contratan temporeros. Mantienen niveles de mecanización similares a las explotaciones más pequeñas, adecuadas, eso sí, a su tamaño.

En general, si analizamos la relación que se produce entre el número de trabajadores y la proporción entre ellos de fijos y temporales, constatamos que en la mayoría de las explotaciones predomina la plantilla de temporeros.

La poda (23\%), el aclareo (25\%) y la recogida $(26,5 \%)$ son las actividades predominantes que realiza el personal fijo, puesto que son, a pesar de las innovaciones técnicas y mecánicas incorporadas, las actividades que requieren más mano de obra, tanto de los trabajadores fijos y de la unidad familiar de explotación (en las explotaciones de menor tamaño), como de los contratados temporalmente. El personal fijo también lleva a cabo las tareas que son más regulares en el ciclo productivo, que requieren a pesar de las mejoras técnicas introducidas en el instrumental y la maquinaria, su manipulación por personas en un porcentaje de tiempo importante, respecto otras actividades que necesita la producción frutera, y que no responden a picos intensificados de necesidad de mano de obra, como puede ser la aplicación de fitosanitarios, herbicidas, segar las hierbas o regar los frutales con dispositivos de gota a gota, aunque este tipo de riego suele estar programado con dispositivos ofimáticos.

Debemos remarcar que el coste de la poda, el aclareo y la recogida de la fruta representan un porcentaje fijo en el coste del producto final, que hoy por hoy, junto a los tratamientos fitosanitarios y el riego, parece muy difícil de disminuir. Incluso se produce la paradoja que en algunas campañas, y especialmente para las variedades de menor demanda, estos costes pueden ser más altos que la cantidad dineraria que los fruticultores reciben por estos productos.

La participación de los temporeros en los trabajos frutícolas se produce para actividades y momentos del ciclo productivo muy concretos, y en este orden descendiente: recogida de la fruta, aclareo y poda. En este sentido, se ha producido un importante cambio respecto a la época de difusión de las plantaciones de frutales entre los años 60 y 70 del pasado siglo, cuando las explotaciones fruteras 
eran de menor tamaño. Entonces casi todas las actividades de poda las llevaban a cabo las mismas unidades familiares, ayudadas en algunos casos, por la contratación de podadores especializados, todos autóctonos. El aclareo y la recogida eran también una actividad de la unidad familiar, con el apoyo de jóvenes estudiantes y temporeros autóctonos. En las últimas décadas, los trabajadores temporeros, la mayoría inmigrantes, acaparan los trabajos de aclareo y recogida, y más recientemente se están incorporando a las actividades de poda, que por otra parte están sufriendo una importante mecanización, por lo que se ha reducido el tiempo dedicado a ello de forma manual (aunque no en todas las variedades y tipos de formación de los árboles frutales).

\subsection{Los trabajadores de las explotaciones según los empre- sarios}

La mayoría de los agricultores (77\%) contrata siempre a los mismos temporeros y lo justifican porque valoran, y en este orden, la comodidad de hacerlo así (40\%), la confianza en ellos puesto que los conocen (41\%) y porque saben que trabajan bien (61\%). El resto que no contrata a los mismos temporeros, es porque estos en años venideros ya no vuelven. Lo cual nos lleva a poder afirmar que el nivel de satisfacción de los ocupadores es bastante alto respecto al trabajo de los temporeros y que prefieren mantener con ellos una relación laboral, aunque temporal, más dilatada, ya que así pueden disponer de ellos en los momentos de mayor necesidad de mano de obra, y porque al conocer su comportamiento y rendimiento, consideran que es una opción acertada. Estos datos confirman el esfuerzo hecho por sus organizaciones empresariales y por otras instituciones, en el sentido de asentar temporeros en el territorio, con la construcción de viviendas o espacios de acogida para su residencia más o menos estable, bajo la forma del alquiler u otras, o bien mediante la rehabilitación de viviendas de alquiler.

Los temporeros llevan a cabo jornadas medias de trabajo de entre 8 y 9 horas, así como los trabajadores estables. El horario está en función de la época del año y de las condiciones climatológicas, alargándose las jornadas de trabajo en primavera (aclareo) y en verano y principios de otoño (recolección).

La mitad de los trabajadores fijos suelen trabajar cinco días a la semana, por contra los temporeros suelen trabajar seis, coincidiendo con los picos de trabajo manual que se producen regularmente en los momentos del aclareo y recolección, que deben realizarse dentro de unos marcos de tiempo muy concretos. Algunas de las labores que realizan los trabajadores fijos a lo largo del año pueden planificarse y distribuirse en un horario semanal de cinco días solamente, por contra 
durante el aclareo y especialmente en la recolección los márgenes de tiempo para realizar las tareas son más reducidos.

La mayoría de los agricultores encuestados (71\%) tiene picos o momentos de mucho trabajo, debido al ciclo de la naturaleza que determina las labores a realizar en los frutales, pero, además, como decimos en el anterior apartado, el aclareo y la recolección se llevan a cabo en unos períodos o márgenes de tiempo que no se pueden alargar, puesto que comportaría perder parte de la producción, por un exceso de fruta que repercutiría en una disminución del calibre requerido para su comercialización; o porque maduraría más de lo que suele ser aconsejable para su manipulación, selección y transporte a los lugares de venta o a las cámaras de frío regulado para su conservación.

La contratación de trabajadores por parte de los agricultores, su número y proporción entre fijos y temporeros responde a los trabajos que hay que realizar a lo largo de una cosecha, aunque los temporeros se contratan cuando se producen esos momentos de una mayor demanda de mano de obra, que suele ser muy variable según el tipo de fruta que se produzca, y los porcentajes de cada variedad, lo que determina épocas de aclareo distintas, y sobre todo de recolección.

La mayoría de agricultores resuelven estos picos de trabajo y se adecuan a ellos contratando más trabajadores, produciéndose oscilaciones en cada explotación frutera del número de los que se contratan como temporeros, casi a un ritmo de semana a semana, en función de las labores y variedad de fruta para la cual se requieren. Un 50\% también lo resuelve alargando las jornadas mediante horas extraordinarias. Ambas estrategias no son opuestas sino complementarias, y conviven ambas en la misma explotación, en función de los trabajos a realizar, la rapidez en que se necesita realizarlos y la disponibilidad de temporeros.

Cuando, por el contrario, los agricultores tienen una bajada de la necesidad de trabajo en sus explotaciones, responden a la misma dejando de contratar temporeros, o en menor medida encargándoles otros trabajos.

La mayoría de los agricultores contratan directamente a sus trabajadores, ya que como hemos visto anteriormente, la mayoría prefiere contratar trabajadores que ya conocen. Cuando no pueden hacerlo así, porque los temporeros no vuelven al año siguiente, utilizan otras vías, y entre ellas, la dominante es mediante contactos informales, que suponemos les proporcionan otros productores, trabajadores temporeros o conocidos. En este punto debemos resaltar que casi son inexistentes los contratos mediante las bolsas de trabajo, tanto públicas como privadas, así como mediante los sindicatos y organizaciones agrarias. En el estudio no hemos detectado ningún caso de contratación en origen. 
Los agricultores manifiestan que estos procedimientos utilizados como vía de contratación llevan tiempo realizándose (más de 8 años de media), y que no se han producido cambios, ni parece que se vayan a producir en un futuro.

Cuando se les pregunta a los agricultores sobre la forma de incrementar la contratación de trabajadores estables y temporales, optan por posicionarse en la posibilidad de incrementar el tamaño de la explotación con más hectáreas disponibles, o mediante la intensificación de la producción. Choca que se posicionen de esta forma, ya que con las estructuras existentes, parece difícil cambiar estos dos elementos sin una capitalización importante, que permita comprar o alquilar tierras o introducir mejoras que suelen tener un coste importante para ellos, como son las redes para protegerse del pedrisco, o la mejora y eficiencia de los riegos, o los sistemas para hacer frente a las heladas tardías en época de floración. Solamente una minoría concreta la mejora del precio de la fruta como alternativa.

Cuando los agricultores contratan trabajadores valoran en orden de menor a mayor: que sean responsables, que hayan demostrado ser de confianza y especialmente que sean buenos trabajadores. También valoran que no se quejen, que se tomen el trabajo en serio y que sean responsables.

No resaltan experiencias negativas, excepto en pocos casos, en que señalan como justificación la poca profesionalidad de los temporeros contratados, o los conflictos por la diversidad de los orígenes de los contratados. Solamente un 10\% remarca que porque los considera conflictivos. Todo ello es compatible con la afirmación de que la mayoría de agricultores vuelve a contratar los temporeros que previamente ya habían contratado.

Casi todos los ocupadores prefieren para todas las actividades que contratan trabajadores, que estos sean hombres. El intervalo de edad preferido, aunque sin mucha diferencia con los otros intervalos de edad, es el de 25 a 34 años. En el imaginario social de los agricultores predomina la idea de que las labores del campo, en particular las relacionadas con la producción frutera son desarrolladas de manera más efectiva por el sexo masculino, relacionado tal vez con las condiciones de trabajo al aire libre y con la fuerza que algunas de estas tareas requieren. Por el contrario para la manipulación de la fruta y su empaquetado prefieren a las mujeres. Lo que nos lleva a reflexionar sobre las diferencias de género existentes en el sector de la fruta, que están relacionas con los estereotipos sobre los hombres y las mujeres.

Preguntados los agricultores sobre sus preferencia por el origen de sus trabajadores y relacionado con las diferentes actividades de la producción y manipulación de la fruta, cabe destacar que en todas ellas prefieren mayoritariamente a los trabajadores de origen subsahariano, seguidos de los catalanes y de los del resto 
del estado español. No tenemos ninguna interpretación para este posicionamiento más allá de la influencia de los estereotipos que relacionan a los trabajadores con cualidades relativas a su origen o procedencia étnica.

Respecto al precio por hora de trabajo y las diferencias existentes entre trabajadores temporeros y fijos, la media que perciben los temporeros es de 5,77€ por hora trabajada, y de 5,83€ para los trabajadores fijos, lo cual evidencia que no existen diferencias importantes entre los trabajadores del sector, sean estos fijos o temporeros.

Casi todos los trabajadores fijos cobran su sueldo a finales de mes y los temporeros lo hacen mayoritariamente cada semana. Diferencias que tienen que ver con la duración de los contratos, ya que no siempre, los temporeros se contratan por períodos mensuales, sino en función de los picos de trabajo, que pueden ser relativamente cortos (semana, quincena). En todo caso el 37\% de los temporeros que cobran a finales de mes puede ser un indicador más o menos preciso de los porcentajes de temporeros que se contratan por períodos iguales o más largos de un mes.

Solamente un $2 \%$ de los ocupadores manifiesta haber contratado trabajadores estables sin contrato, porcentaje que aumenta al $25 \%$ en el caso de los temporeros. Por tanto, los agricultores no suelen ocupar trabajadores sin contrato, excepto en casos de puntas de trabajo, que es la razón que aducen para realizarlo.

Casi la mitad de los agricultores ofrecen algún servicio a los trabajadores, siendo la vivienda el más importante entre los que ofrecen (91\%) y en menor medida el transporte (36\%). Hay que tener en cuenta que la mayoría de los trabajadores se desplazan a las parcelas por cuenta del ocupador (73\%).

La mayoría de los ocupadores conoce la existencia y la práctica de los intermediarios para contratar a trabajadores temporeros, pero muy pocos lo consideran útil. Un $28 \%$ considera que las ONGs son necesarias para la contratación y gestión de la campaña agrícola, ya hemos visto que la mayoría contratan a trabajadores que ya conocen de otras campañas, y que cuando no lo hacen es porque los trabajadores no regresan en la nueva campaña.

Respecto a las inspecciones de trabajo el $60 \%$ de los ocupadores están de acuerdo en que son necesarias, aunque una tercera parte considera que dificultan las cosas y una minoría creen que no deberían existir. No hay un acuerdo unánime sobre si han aumentado o disminuido. Sí que se perciben quejas sobre la cuantía de las sanciones económicas ya que las consideran altas.

Respecto la contratación en origen, solamente un 40\% está a favor, aunque nadie la utiliza. Aducen como motivos a su favor: la adaptación al calendario de trabajo y que asumen las necesidades de la empresa. Los contrarios creen que 
hay suficiente mano de obra aquí. La mayoría de los agricultores están en contra de contratar personas sin papeles, por los riesgos laborales que tal modalidad comporta.

\section{Conclusiones}

La mayoría de los encuestados se dedican a actividades relacionadas con la agricultura y, dentro de éstas, a la explotación de árboles frutales, siendo la media de las explotaciones de 20ha. En periodos estacionales contratan temporeros, mayormente hombres jóvenes para tareas de recogida, aclareo y poda de árboles.

Su primera opción es contratar, si es posible, los mismos trabajadores que en la anterior campaña. Entre las motivaciones que argumentan para esta postura figuran: el hecho de que ya los conocen (saben cómo trabajan y en cierta forma qué tipo de persones son frente al trabajo y la convivencia de grupo); el hecho de que el empresario se ahorra toda la tarea que significa iniciar los trámites de búsqueda de nuevos trabajadores y finalmente el hecho de que aquellos ya conocen las tareas que deben realizarse en la explotación agrícola (no será necesario enseñarles).

Si se produce "cambios" de trabajadores será mayormente imputable a que éstos "deciden no volver" o no aparecen cuando se les espera, no por causas imputables a los propios empresarios.

Los empresarios utilizan muy poco las empresas de intermediación y optan, de forma muy mayoritaria, por utilizar los servicios que les ofrecen los Sindicatos agrarios como vía para la obtención de trabajadores temporeros.

Los ocupadores afirman, de forma muy mayoritaria, que optan por regularizar en todos los casos de forma legal las contrataciones y niegan que, en el sector agrario se produzcan contrataciones irregulares o trabajo de economía sumergida. Razonan esta afirmación en base a los riesgos legales que comporta esta práctica y por encontrarse fuera de la regulación laboral vigente.

A partir de las entrevistas, podemos concluir que la organización y planificación de las campañas surge del intento de evitar las situaciones problemáticas que tenían lugar cuando las campañas agrícolas comenzaron a masificarse. Todo apunta a que el eje conductor de las organizaciones de empresarios es asegurar que las personas que acuden a la campaña de la fruta ilerdense sean sólo aquellas que ya tienen pactado un trabajo antes de llegar.

Con ello, según su punto de vista, se busca evitar grandes colectivos de solicitantes de trabajo rondando la región durante los meses estivales como pasaba en los años anteriores a que la planificación de la campaña estuviese tan estructurada. Así, se ha conseguido reducir considerablemente las dos problemáticas 
más vinculadas a la campaña de la fruta: los campamentos y las contrataciones irregulares.

Asimismo, hemos podido constatar que los sindicatos agrícolas dan gran importancia a la búsqueda de estabilidad de sus trabajadores a lo largo del año. Pese a no ser uno de los medios más usados, todos ellos defienden la importancia de sus bolsas de trabajo para ayudar a mejorar la calidad de vida de los temporeros.

\section{Bibliografía}

Achón Rodríguez, Olga (2010). "Contratación en origen e institución total. Estudio sobre el sistema de alojamiento de trabajadores agrícolas extranjeros en el Segrià" (tesis doctoral). Universitat de Barcelona, España.

Català Rubio, Santiago (2013). "La crisis económica española vista desde la otra orilla". Encuentros multidisciplinares, 15(43): 72-80.

Díaz Diego, José (2009): "Los campos que otros trabajan. Las campañas agrícolas españolas con mayor porcentaje de extranjeros", en Gordo Márquez, Mercedes y García, Felicidades (Coord.) (2009). Explorando los contratos en origen en los campos españoles. Huelva: Universidad de Huelva.

Gracia Royo, A. y Albisu Aguado, L.M. (2004). "Evolución de la industria agroalimentaria española en las dos últimas décadas”. Economía industrial, 355: $197-210$.

Gordo Márquez, Mercedes; Allepuz Capdevila, Rafael; Márquez Domínguez , Juan Antonio y Torres Solé, Teresa (2015). “La gestión colectiva de los contratos en origen de temporeros colombianos en la provincia de Lleida". Boletín de la Asociación de Geógrafos Españoles, 68: 233-252.

López, Isidro y Rodríguez, Emanuel (2010). Fin de siglo, financiarización, territorio y sociedad de propietarios en la onda larga del capitalismo bispano (1959-2010). Madrid, Traficantes de sueños.

López-Sala, Ana; Molinero Gerbeau, Yoan; Jolivet-Guetta, Mélanie; Eremenko, Tatiana; Beauchemin, Cris; Samuk, Sahizer y Consterdine, Erica (2016). "Seasonal immigrant workers and programs in UK, France, Spain and Italy", Working Paper en Temporary versus Permanent Migration 2016/01, TEMPER EU Project.

Márquez Domínguez, Juan Antonio (2009). “Jornaleros forasteros y extranjeros en la frontera agraria”, en Gordo Márquez, Mercedes y FelicidaDES, Jesús (Coord.) (2009). Explorando los contratos en origen en los campos españoles. Huelva: Universidad de Huelva. 
Pascual Roca, Miquel (2010). "La fructicultura a Lleida". En Daniel Baвot et alii, (Coord.) (2010). L'Agricultura a les Terres de Lleida en el segle XXI. Lleida: La Mañana.

Pedreño Cánovas, Andrés (1998). «Del jornalero agrícola al obrero de las factorías vegetales. Estrategias familiares y nomadismo en la ruralidad murciana» (tesis doctoral). Universidad de Murcia, España.

Requeijo González, J. (2005). “La era del Quantum: 1960-1974”. Información Comercial Española, ICE: Revista de economía, 826: 25-37.

Requena, M. (2008). "Bases demográficas dela sociedad española". En: GonzÁlez, J.J., y Requena, M. (ed.) Tres décadas de cambio social en España. Madrid, Alianza: 141-164

TABARes, Esteban (1990): Jornaleros y Temporeros. Madrid: Cáritas Española.

Torres Solé, Teresa; Allepuz Capdevila, Rafael y Gordo Márquez, Mercedes (2013). "La contratación de mano de obra temporal en la agricultura hortofrutícola española". Revista de Estudios sobre Despoblación y Desarrollo Rural, 16:7-37.

Velasco Castro, Antonio José (2009). "Epistemología de la representación del cambio social”. Episteme, 29 (2): 114-144. 